\title{
A comparison of emergency department presentations for medically unexplained symptoms in frequent attenders during COVID-19
}

\author{
Authors: Natasha F Daniels, ${ }^{\mathrm{A}}$ Raiiq Ridwan, ${ }^{\mathrm{B}}$ Ed BG Barnard, ${ }^{\mathrm{C}}$ Talha M Amanullah ${ }^{\mathrm{D}}$ and \\ Catherine Hayhurst ${ }^{\mathrm{E}}$
}

\begin{abstract}
Medically unexplained symptoms (MUS) are those with no identified organic aetiology. Our emergency department (ED) perceived an increase in MUS frequency during COVID-19. The primary aim was to compare MUS incidence in frequent attenders (FAs) during COVID-19 and a control period.

A retrospective list of FA-MUS presenting to our ED from March to June 2019 (control) and March to June 2020 (during COVID-19) was compared. Fisher's exact test was used to compare binomial proportions; this presented as relative risk (RR) with $95 \%$ confidence intervals ( $95 \% \mathrm{CI})$.

During COVID-19, ED attendances reduced by $32.7 \%$, with a significant increase in the incidence of FA-MUS and FAMUS ED visits compared to control; RR 1.5 (95\%CI 1.1-1.8) $\mathrm{p}=0.0006$, and RR 1.8 (95\%CI 1.6-2.0), $\mathrm{p}<0.0001$, respectively.

Despite reduced ED attendances during COVID-19, there was a significant increase in the incidence of FA-MUS patients and corresponding ED visits by this cohort. This presents a challenge to ED clinicians who may feel underprepared to manage these patients effectively.
\end{abstract}

KEYWORDS: COVID-19, medically unexplained symptoms, emergency department, SARS-CoV2

DOI: $10.7861 /$ clinmed.2020-1093

\section{Introduction}

Since the start of the COVID-19 pandemic, the physical and psychological sequelae associated with SARS-CoV-2 are still being discovered. The pandemic's impact on mental health has been

Authors: Afinal year medical student, University of Cambridge School of Clinical Medicine, Cambridge, UK; ${ }^{B}$ specialty trainee in emergency medicine, Cambridge University Hospitals NHS Foundation Trust, Cambridge, UK; ' Consultant in emergency medicine, Royal Centre for Defence Medicine (Research \& Clinical Innovation), Birmingham, UK and Cambridge University Hospitals NHS Foundation Trust, Cambridge, UK; Djunior clinical fellow in emergency medicine, Cambridge University Hospitals NHS Foundation Trust, Cambridge, UK; ${ }^{E}$ Consultant in emergency medicine, Cambridge University Hospitals NHS Foundation Trust, Cambridge, UK extensively discussed in the literature, with admissions for COVID-19related health anxiety described. 1 Particularly challenging emergency department (ED) presentations are those in which symptoms have no identified organic aetiology, referred to as medically unexplained symptoms (MUS) or functional symptoms. ${ }^{2}$ (The term MUS is used here.) These symptoms include non-cardiac chest pain, gastrointestinal complaints, non-epileptic seizures, functional neurology, and shortness of breath (SOB). One European study reported that MUS in the ED were present in $13.4 \%$ of all visits pre-COVID. ${ }^{3}$

MUS is a common presentation in frequent attenders (FAs). In a previously published dataset from our hospital, $45 \%$ of FAs were identified as having one or more MUS. ${ }^{4}$ The most frequently used definition for an FA is a person who attends an ED five times or more in a year. ${ }^{4}$ The impact of MUS on patients can be debilitating, with added stressors due to stigma experienced both within society and the healthcare system. ${ }^{5}$ As well as the personal burden for patients, there is a disproportionate use of healthcare resources by FAs. ${ }^{6,7}$ Patients attend frequently as despite being reassured their condition is not a myocardial infarction, pulmonary embolism, seizure or similarly serious diagnosis, they are not given an adequate explanation for their symptoms. Their repeated attendance may lead clinicians to undertake further investigation, thus adding to cost and increasing the patient's dependence on the health service as well as their risk of iatrogenic harm. ${ }^{8,9}$

Clinicians in our hospital perceived that there was an increase in patients attending with MUS, both among FAs and the general patient population. However, as we routinely collect FA data in our ED, we decided to use FA-MUS as a proxy for the general MUS population. Our objective was to test the perceived hypothesis that MUS in FA incidence had increased during COVID-19.

\section{Methods}

\section{Setting}

Cambridge University Hospitals NHS Foundation Trust (CUH) is a major trauma centre and tertiary referral centre with an ED attendance $>120 \mathrm{k}$ per year. In addition to its specialist roles, $\mathrm{CUH}$ serves the local population (Cambridge, South Cambridgeshire, East Cambridgeshire) as a local ED. Census data were obtained from the Office of National Statistics (ONS) to estimate the local population at risk during the study period (373,725 people, mid-2019) in order to estimate incidence. ${ }^{10}$ 


\section{Study design}

CUH ED attendance data were used to identify FA, defined as five or more ED visits during either 122-day study period: 1 March to 30 June 2019 (control) and 1 March to 30 June 2020 (COVID-19). Two authors independently reviewed all ED case notes for FA patients, and initially categorised visits as physical health, mental health (overdose, deliberate self-harm, psychosis, etc) or MUS. The presenting complaint was designated as MUS if there were investigations with no abnormal findings and no signs of organic disease. The MUS attendances were then independently assessed by the same two authors to categorise them into one of seven presenting complaints: non-cardiac chest pain, abdominal pain, functional neurological symptoms, non-epileptiform seizures, musculoskeletal symptoms, SOB, and other. Any disagreement in either MUS diagnosis or categorisation was arbitrated by a third author.

\section{Primary and secondary aims}

The primary aim was to compare the incidence of FA-MUS during COVID-19 with the control period. The secondary aim was to compare the frequency of MUS presenting complaints between the two time periods.

\section{Ethical review}

This project was registered at CUH as a service evaluation (ID3270 PRN9270); ethical review was not required by the local research ethics committee. Data were handled in accordance with the UK Data Protection Act 2018.

\section{Data analysis}

Data were analysed in Prism for macOS (v.9.0.1 GraphPad Software, San Diego, CA, USA), using the software's recommended statistical tests. Fisher's exact test was used to compare proportions (incidence), and the results are presented as relative risk (RR) with Koopman asymptotic score $95 \%$ confidence intervals $(95 \% \mathrm{CI}$ ) and exact $p$-values (when $p \geq 0.0001)$. In order to report population incidence per 1,000 people per year, the number of patients and the number of visits have been multiplied by 2.99 ( 356.25 days/122 days) and then divided by the ONS local population estimate. A $p$-value $<0.05$ has been considered significant.

\section{Results}

There were a total of $n=42,785 \mathrm{ED}$ visits in the control period and $n=28,806$ in the COVID-19 period, a decrease of $32.7 \%$.

\section{Number of all FA patients and FA visits}

There was no significant difference between the incidence of FA ED patients in the COVID-19 and control periods: RR 0.95 (95\% CI $0.90-1.01), p=0.13$. However, overall there was a significantly lower incidence of ED visits by this cohort in the COVID-19 period, RR 0.92 (95\% CI 0.90-0.94), $p<0.0001$ (see Table 1).

\section{FA-MUS patients and visits}

There was a significant increase in both the incidence of FA-MUS ED patients and the corresponding incidence of FA-MUS ED visits during the COVID-19 period compared to control: RR 1.5 (95\% CI $1.1-1.8), p=0.0006$, and RR 1.8 (95\% CI 1.6-2.0), $p<0.0001$, respectively (see Table 1 ).

The numbers of visits and patients are also represented graphically in Fig 1 to demonstrate the differences between each year.

\section{MUS categories}

FA-MUS visits were categorised into common syndromes based on presenting symptoms; categories with less than ten visits were grouped into 'other' (for example, palpitation $(n=9)$, falls $(n=6)$,

Table 1. A comparison of emergency department overall attendances, frequent attenders, and frequent attenders with medically unexplained symptoms between two 122-day periods in 2019 (Control) and 2020 (COVID-19)

\begin{tabular}{|c|c|c|c|}
\hline & Control & COVID-19 & RR (95\%CI), p-value \\
\hline ED visits, $n$ & 42,785 & 28,806 & \\
\hline ED visits per $1 \mathrm{kpop} / \mathrm{yr}$ & 114.4 & 77.0 & \\
\hline \multicolumn{4}{|l|}{ Frequent attenders } \\
\hline Patients, $\mathrm{n}$ & 163 & 147 & \\
\hline Patients per $1 \mathrm{kpop} / \mathrm{yr}$ & 1.3 & 1.2 & 0.95 (95\% CI 0.90-1.01), $p=0.13$ \\
\hline ED visits, $n$ & 1185 & 1000 & \\
\hline ED visits per $1 \mathrm{kpop} / \mathrm{yr}$ & 9.5 & 8.0 & $0.92(95 \%$ CI $0.90-0.94), p<0.0001$ \\
\hline \multicolumn{4}{|c|}{ Frequent attenders with medically unexplained symptoms } \\
\hline Patients, n & 44 & 65 & \\
\hline Patients per $1 \mathrm{kpop} / \mathrm{yr}$ & 0.4 & 0.5 & $1.5(95 \%$ CI 1.1-1.8), $p=0.0006$ \\
\hline ED visits, $n$ & 149 & 267 & \\
\hline ED visits per $1 \mathrm{kpop} / \mathrm{yr}$ & 1.2 & 2.1 & 1.8 (95\% CI 1.6-2.0), $p<0.0001$ \\
\hline
\end{tabular}




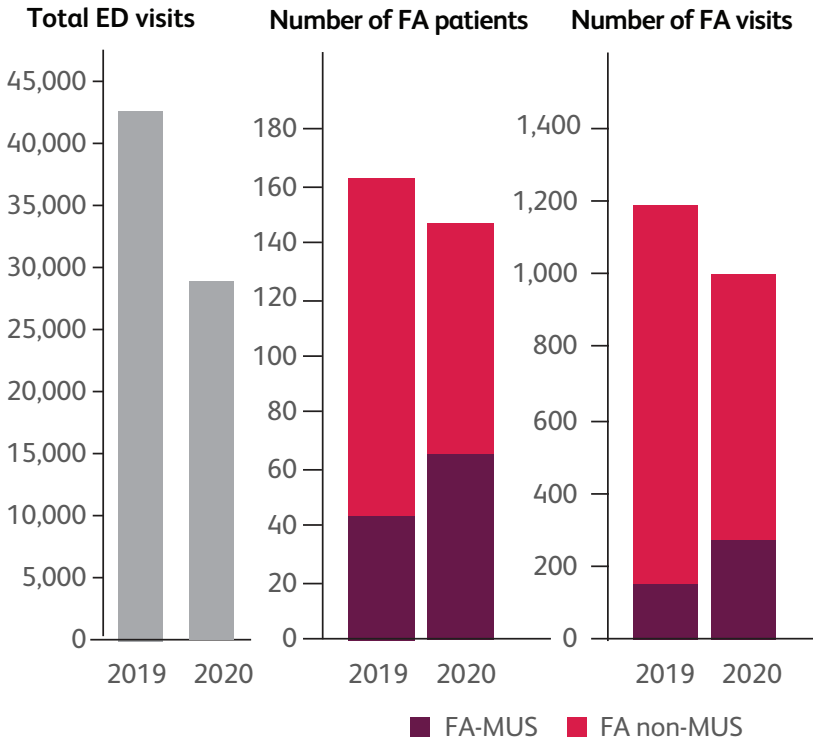

Fig 1. Bar charts showing the total number of ED attendances, number of FA patients and number of FA visits between March to June in 2019 (control) and in 2020 (the COVID-19 period). ED = emergency department, $F A=$ frequent attender, FA-MUS = frequent attender with medically unexplained symptoms, FA non-MUS = frequent attender with a presentation that was not MUS.

vomiting $(n=5))$. The only difference observed was a significant increase in the MUS category SOB during the COVID-19 period (Table 2).

\section{Discussion}

This study has demonstrated a significant increase in the incidence of FA-MUS attendances to our ED during COVID-19, a phenomenon previously reported in other cases of unpredictable

Table 2. The frequency of medically unexplained symptom presenting complaint categories in frequent emergency department attenders between two 122-day time periods in 2019 (control) and 2020 (COVID-19)

\begin{tabular}{llll} 
MUS diagnosis & Control & COVID-19 & p-value \\
$\begin{array}{l}\text { Non-cardiac chest pain, } \\
\mathrm{n}(\%)\end{array}$ & $72(48.3 \%)$ & $117(43.8 \%)$ & 0.41 \\
Abdominal pain, $\mathrm{n}(\%)$ & $35(23.5 \%)$ & $56(21.0 \%)$ & 0.62 \\
Neurological, $\mathrm{n}(\%)$ & $13(8.7 \%)$ & $33(12.4 \%)$ & 0.33 \\
$\begin{array}{l}\text { Non-epileptiform seizure, } \\
\mathrm{n}(\%)\end{array}$ & $12(8.1 \%)$ & $16(6.0 \%)$ & 0.42 \\
Musculoskeletal, $\mathrm{n}(\%)$ & $3(2.0 \%)$ & $15(5.6 \%)$ & 0.13 \\
Shortness of breath, $\mathrm{n}(\%)$ & 0 & $11(4.1 \%)$ & $<0.01$ \\
Other, $\mathrm{n}$ & 14 & 19 & \\
Total, $\mathrm{n}$ & 149 & 267 & \\
\hline
\end{tabular}

MUS = medically unexplained symptoms threats. ${ }^{11}$ The implications of this are that despite the risk of attending an ED in the context of a pandemic, these symptoms are debilitating enough for the patients to deem the risk of a hospital visit necessary. It emphasises how distressful MUS are, to the extent that the risk of contracting a potentially deadly disease does not trump their perceived need for investigation. There are no referral pathways or secondary care psychology services from the ED to aid patients with MUS, leaving ED staff with limited resources to help this population. Additionally, as COVID-19 is more likely to be a long-term phenomenon, it is important to emphasise the impact of this disease on the MUS population, and the provision of services for ED staff to better manage this population.

SOB was the only MUS presentation that significantly increased during COVID-19, likely secondary to pandemic-related anxiety. It is perhaps unsurprising that non-cardiac chest pain was the commonest type of MUS seen in our ED, as patients worry about having cardiac disease. ${ }^{12}$ Patients with MUS-type SOB are seen in the ED but are usually in patients who already have pre-existing lung conditions, eg COPD (chronic obstructive pulmonary disease), or they do not present frequently enough to reach the definition of a frequent attender. During COVID-19, patients presented frequently with $\mathrm{SOB}$ with no identified physical cause.

To the best of our knowledge, there are no studies examining how the COVID-19 pandemic has influenced this population. MUS are poorly understood, but it is accepted that these symptoms are real, have no identifiable underlying pathology, and may be brought about by a physical or psychological trigger. ${ }^{13}$ Symptomology can become reinforced by repeated healthcare encounters. Added to the stress and anxiety felt by the majority of the population during the first wave of COVID-19,14 it is probable that novel neuropsychological pathways were more likely to be formed during this pandemic.

Generally, the default management of the MUS patient is extensive investigation to rule out physical health pathology, followed by psychiatric assessment and/or discharge. However, it has been reported that early diagnosis with reassurance and an explanation regarding the mechanisms of such symptoms can be of benefit. ${ }^{5}$ Alternatively, a stepped psychological approach may be utilised..$^{15,16}$

ED providers often report uncertainty in managing patients with MUS, indicating a need for training so that investigations to rule out other pathology are balanced with early diagnoses and appropriate interventions. Some of these patients may be better served within primary care, but this depends on the ease of access and primary care clinicians being confident in MUS diagnosis and management.

The high prevalence of FA-MUS in the ED is likely a symptom of the general trend of unmet needs for this diverse and vulnerable group elsewhere in the healthcare system. This paper adds further evidence that the needs of these individuals with MUS may not be being met, and in added stressors such as the COVID-19 pandemic, their needs are further exacerbated.

\section{Limitations}

The data analysis was performed retrospectively and represents a single ED and thus the results are not necessarily translatable to other centres. Additionally, due to the perceived increase in frequency of MUS patients before commencing the data extraction, reviewer bias is an important consideration. This was minimised by having two reviewers (ND and RR) extract the data from both 2019 and 2020. There was no blinding due to a need 
to detect those that were FA within a specific timeframe, further limiting the results. Confirmation bias must also be considered given the lack of a clear universal definition of MUS. However, this was minimised by discussing ambiguous cases between the reviewers. Finally, due to the observational nature of the study, there are potentially unobserved confounding variables that could have influenced the results other than COVID-19. The characteristics of the FA between periods may have differed and thus direct causal connections should not be drawn.

\section{Conclusion}

While the total number of ED attendances reduced by almost one third during COVID-19, the incidence of all visits by FA-MUS increased during the same period. This paper highlights the significance of the MUS experience, with patients willing to risk their safety at the peak of the pandemic. This speaks volumes of the severity of the FA-MUS patient experience and should prompt the general healthcare system to consider how to better manage this patient group.

\section{Summary}

\section{What is known about the topic?}

Medically unexplained symptoms (MUS) are those that have no identified organic aetiology. They are a heterogeneous set of conditions that have biopsychosocial factors and are among the most challenging presentations for patients and ED staff.

\section{What question did this study address?}

Was there an increase in the incidence of MUS presentations among frequent attenders (FA-MUS) during the COVID-19 pandemic?

\section{What did this study find?}

There was a significant increase in both the incidence of FA-MUS patients and the corresponding incidence of FA-MUS ED visits during the COVID-19 period compared to a date-matched historical control period.

\section{What is the implication for practice now?}

This reinforces our need as clinicians to be able to manage these patients well and emphasises that despite the risk of attending an ED in the context of a pandemic, these symptoms are debilitating enough for patients to deem the risk of a hospital visit necessary.

\section{References}

1 Asmundson G], Taylor S. How health anxiety influences responses to viral outbreaks like COVID-19: what all decision-makers, health authorities, and health care professionals need to know. J Anxiety Disord 2020:71:102211.
2 Rosendal M, Hartman TC, Aamland A et al. 'Medically unexplained' symptoms and symptom disorders in primary care: prognosis-based recognition and classification. BMC Fam Pract 2017;18:18.

3 Alsma J, van de Wouw J, Jellema K et al. Medically unexplained physical symptoms in patients visiting the emergency department: an international multicentre retrospective study. Eur ] Emerg Med 2019;26:249-54.

4 Jacob R, Wong ML, Hayhurst C, Watson P, Morrison C. Designing services for frequent attenders to the emergency department: $a$ characterisation of this population to inform service design. Clin Med 2016;16:325-9.

5 Sowiska A, Czachowski S. Patients' experiences of living with medically unexplained symptoms (MUS): a qualitative study. BMC Fam Pract 2018:19:23.

6 Burton C, McGorm K, Richardson G, Weller D, Sharpe M. Healthcare costs incurred by patients repeatedly referred to secondary medical care with medically unexplained symptoms: a cost of illness study. J Psychosom Res 2012;72:242-7.

7 Greenfield G, Blair M, Aylin PP et al. Frequent attendances at emergency departments in England. Emerg Med J 2020;37:597-9.

8 Warner A, Walters K, Lamahewa K, Buszewicz M. How do hospital doctors manage patients with medically unexplained symptoms: a qualitative study of physicians. J R Soc Med 2017;110:65-72.

9 Hatcher S, Arroll B. Assessment and management of medically unexplained symptoms. BMJ 2008;336:1124-8.

10 Office for National Statistics. Middle Super Output Area population estimates (supporting information). www.ons.gov.uk/peoplepopula tionandcommunity/populationandmigration/populationestimates/ datasets/middlesuperoutputareamidyearpopulationestimates [Accessed 6 March 2021].

11 Guerriero RM, Pier DB, De Gusmão CM et al. Increased pediatric functional neurological symptom disorders after the Boston marathon bombings: a case series. Pediatric Neurol 2014;51:619-23.

12 Rogers J, Collins G, Husain M, Docherty M. Identifying and managing functional cardiac symptoms. Clin Med 2021;21:37-43.

13 Burton C. Beyond somatisation: a review of the understanding and treatment of medically unexplained physical symptoms (MUPS). $\mathrm{Br}$ J Gen Pract 2003;53:231-9.

14 Salari N, Hosseinian-Far A, Jalali R et al. Prevalence of stress, anxiety, depression among the general population during the COVID-19 pandemic: a systematic review and meta-analysis. Global Health 2020:16:57.

15 Morriss R, Kai J, Atha C et al. Persistent frequent attenders in primary care: costs, reasons for attendance, organisation of care and potential for cognitive behavioural therapeutic intervention. BMC Fam Pract 2012;13:39.

16 Chew-Graham CA, Heyland S, Kingstone T et al. Medically unexplained symptoms: continuing challenges for primary care. $\mathrm{Br}$ ] Gen Pract 2017:67:106-7.

Address for correspondence: Dr Natasha Daniels, School of Clinical Medicine, University of Cambridge, Hills Road, Cambridge, CB2 0SP, UK.

Email: tasha.dan@outlook.com 